\title{
Crosstalk between Red Blood Cells and the Immune System and Its Impact on Atherosclerosis
}

\author{
Brigitta Buttari, Elisabetta Profumo, and Rachele Riganò \\ Department of Infectious, Parasitic and Immune-Mediated Diseases, Istituto Superiore di Sanità, \\ Viale Regina Elena 299, 00161 Rome, Italy \\ Correspondence should be addressed to Rachele Riganò; rachele.rigano@iss.it
}

Received 26 June 2014; Accepted 16 January 2015

Academic Editor: Robin Van Bruggen

Copyright (C) 2015 Brigitta Buttari et al. This is an open access article distributed under the Creative Commons Attribution License, which permits unrestricted use, distribution, and reproduction in any medium, provided the original work is properly cited.

\begin{abstract}
Atherosclerosis is a chronic multifactorial disease of the arterial wall characterized by inflammation, oxidative stress, and immune system activation. Evidence exists on a pathogenic role of oxidized red blood cells (RBCs) accumulated in the lesion after intraplaque hemorrhage. This review reports current knowledge on the impact of oxidative stress in RBC modifications with the surface appearance of senescent signals characterized by reduced expression of CD47 and glycophorin A and higher externalization of phosphatidylserine. The review summarizes findings indicating that oxidized, senescent, or stored RBCs, due to surface antigen modification and release of prooxidant and proinflammatory molecules, exert an impaired modulatory activity on innate and adaptive immune cells and how this activity contributes to atherosclerotic disease. In particular RBCs from patients with atherosclerosis, unlike those from healthy subjects, fail to control lipopolysaccharide-induced DC maturation and T lymphocyte apoptosis. Stored RBCs, accompanied by shedding of extracellular vesicles, stimulate peripheral blood mononuclear cells to release proinflammatory cytokines, augment mitogen-driven $\mathrm{T}$ cell proliferation, and polarize macrophages toward the proinflammatory M1 activation pathway. Collectively, literature data suggest that the crosstalk between RBCs with immune cells represents a novel mechanism by which oxidative stress can contribute to atherosclerotic disease progression and may be exploited for therapeutic interventions.
\end{abstract}

\section{Introduction}

Atherosclerosis is a chronic inflammatory disease of the arterial wall and the leading cause of mortality in the western world [1]. Research over recent years has delineated atherosclerosis as a multifactorial disease because several factors such as hyperlipidemia, hypertension, diabetes mellitus, male gender, obesity, and family history of cardiovascular disease are implicated. Many of these factors promote mechanisms of inflammation and oxidative stress, which are both major characteristics of atherosclerosis which starts as a "response to injury" favoring immune system activation and endothelial dysfunction [1,2]. Immune system, with elements of both innate and adaptive immunity, contributes positively and negatively to the development of complex atherosclerotic plaques. Plaque macrophages, dendritic cells (DCs), and activated $\mathrm{T}$ and $\mathrm{B}$ lymphocytes represent the majority of leukocytes in the atherosclerotic lesions and their secretory activity, including proinflammatory cytokine and matrix-degrading protease release, may be related to plaque rupture [3-5]. In particular, immune cells of both innate and adaptive immunity may be activated by various endogenous molecules that have undergone chemical and/or structural modification following oxidative or glycation processes. In this way the immune system activation gives rise to low level inflammation leading to the slow development of atherosclerotic disease [6].

Intraplaque hemorrhage, a common event in vulnerable atherosclerotic lesions, results in the deposition of red blood cells (RBCs) and release of haemoglobin ( $\mathrm{Hb})$ [7]. The deposition of RBC membranes within atherosclerotic plaque, providing a large amount of lipids, represents a critical event in the plaque instability [8]. Several lines of evidence have led to attention to a possible pathogenic role of oxidized RBCs in atherogenesis, hypertension [9], coronary artery disease [10], and stroke [11, 12]. The crosstalk of RBCs with immune 
system has been investigated in the field of basic physiological processes $[1,2,13-15]$. We have recently provided evidence that oxidized RBCs present different immunomodulatory activities and suggested that these activities may contribute to perpetuation of inflammation in the pathogenesis of atherosclerosis [16, 17].

In this review, we summarize evidence on the involvement of oxidized RBCs in atherosclerotic disease and in particular on the crosstalk of these cells with innate and adaptive immunity, process that is not traditionally associated with the pathogenesis of this disease (Figure 1).

\section{Red Blood Cells from Scavenger to Prooxidant Cells}

Under physiological conditions, RBCs act as circulating scavengers of oxygen and nitrogen reactive species generated in the vasculature through their well-equipped antioxidant machinery such as reduced glutathione, thioredoxin, ascorbic acid, and vitamin E [18]. When RBCs cross a tissue where an intense production of prooxidant reactive species takes place, the RBC defenses get overwhelmed or are unable to counteract the new prooxidant status of the microenvironment and RBCs become themselves a source of reactive oxygen species (ROS) [18].

Oxidative stress causes a plethora of RBC changes among which are cytoskeleton rearrangement and oxidation and loss of lipid asymmetry. These cells become more rigid and, thus, undergo lysis more easily releasing cytotoxic species in the vasculature. Oxidized RBCs release $\mathrm{Hb}$, heme-Fe, and iron $[19,20]$. These molecules are powerful oxidants and sources of radicals and are able to activate in a proinflammatory way endothelial and innate immune cells [21-25], thus contributing to atherosclerotic plaque instability $[24,26]$. Moreover, RBCs, exposed to both endogenous and exogenous sources of ROS, undergo not only mechanical alteration such as decreased deformability but also accumulation of oxidative damage products such as lipid oxidation products [27] and 4-hydroxynonenal [28] that may represent endogenously formed factors capable of triggering vascular inflammation [29].

To the current knowledge, ROS represent an important factor into the generation of powerful senescent signals on RBCs. It should be noted that oxidized RBC is not a synonymous of senescent RBC. RBC oxidation may be involved in RBC aging and oxidized RBCs show the phenotype of senescence. The major feature of the senescent RBCs is the clustering and/or the breakdown of Band 3 including the binding of oxidized $\mathrm{Hb}$ to high affinity sites on Band $3[30,31]$, and the complexation of $\mathrm{Hb}$ with spectrin is also a prominent and probably prior marker of in vivo RBC aging process, tightly correlated with increased RBC rigidity, decreased deformability, echinocytosis, and erythrophagocytosis [32, 33]. Considering that Band 3 modifications and modification of preexisting molecule generating pathways are mostly the downward consequence of oxidative provocations, it is likely that in conditions of distorted homeostasis oxidized RBCs result as product of an "accelerating senescence" pathology associated with the premature appearance of cellular senescence phenotype in younger RBCs.

Regarding other RBC signaling pathways, the loss of glycophorin A [34], the externalization of phosphatidylserine (PS) [35], and the reduction of the "marker of self" integrinassociated protein CD47 expression [36] have been documented in circulating senescent RBCs.

A similar senescence phenotype has been reported for RBCs during the storage period $[37,38]$, with the exception of PS that has not been detected at the external of stored RBC membrane [39]. Refrigerated storage of RBC units for transfusion results in a complex array of physicochemical changes to RBCs, referred to as the storage lesion [40, 41]. A number of the storage-related changes are similar to those seen in senescent or damaged RBCs in vivo and may reduce the survival of RBCs when transfused.

Oxidative modifications of RBCs have been reported in several chronic inflammatory diseases such as atherosclerosis, coronary artery disease, metabolic syndrome, and multiple sclerosis $[10,16,17,42,43]$.

Our research group found alterations in the redox and aging markers of RBCs from patients with carotid atherosclerosis $[16,17]$. Patient RBCs exhibited a senescent phenotype and presented an increase of intracellular reactive oxygen and nitrogen species and a decrease of intracellular reduced thiols with respect to RBCs from healthy donors. Of note, our results highlight an increased appearance of oxidized RBCs in the group of male patients in line with the well-known gender differences detected in cardiovascular disease onset and progression $[44,45]$. Our experimental evidence on the senescent phenotype acquired by RBCs from healthy subjects following in vitro oxidation suggests that in patients with carotid atherosclerosis the appearance of redox and aging markers of RBCs may be ascribed to the high oxidative stress associated with the pathology [17]. High levels of oxidative stress in patients may be generated by atherosclerotic risk factors such as diabetes, smoking, and hypercholesterolemia.

Under oxidative stress or poor glycemic control, RBCs may undergo posttranslational modifications due to nonenzymatic glycosylation (glycation) reaction, a process that leads to the formation of glycated proteins termed advanced glycation end products (AGEs) [46, 47]. The interaction of AGEs with their receptor on endothelial cells or immune cells [48-51] may contribute to vascular perturbation in chronic disorders related to endothelial cell dysfunction such as diabetes and atherosclerosis [52].

Of interest, mouse RBC treated in vitro with the antioxidant N-acetyl-L-cysteine (NAC) and then intravenously injected into the sibling mice have showed a prolonged halflife with respect to untreated RBCs [53]. There is also evidence that RBCs stored under the antioxidant and membranestabilizing effect of mannitol exhibit a different expression pattern of senescence markers [54]. This evidence supports the hypothesis that $\mathrm{RBC}$ oxidation is involved in $\mathrm{RBC}$ senescence and complements the findings on the increased appearance of oxidized RBCs in cardiovascular diseases.

Remarkably, oxidized/senescent RBCs have been proposed as useful biomarkers to monitor oxidative alterations in the progression of chronic or acute diseases [18, 55-57]. 


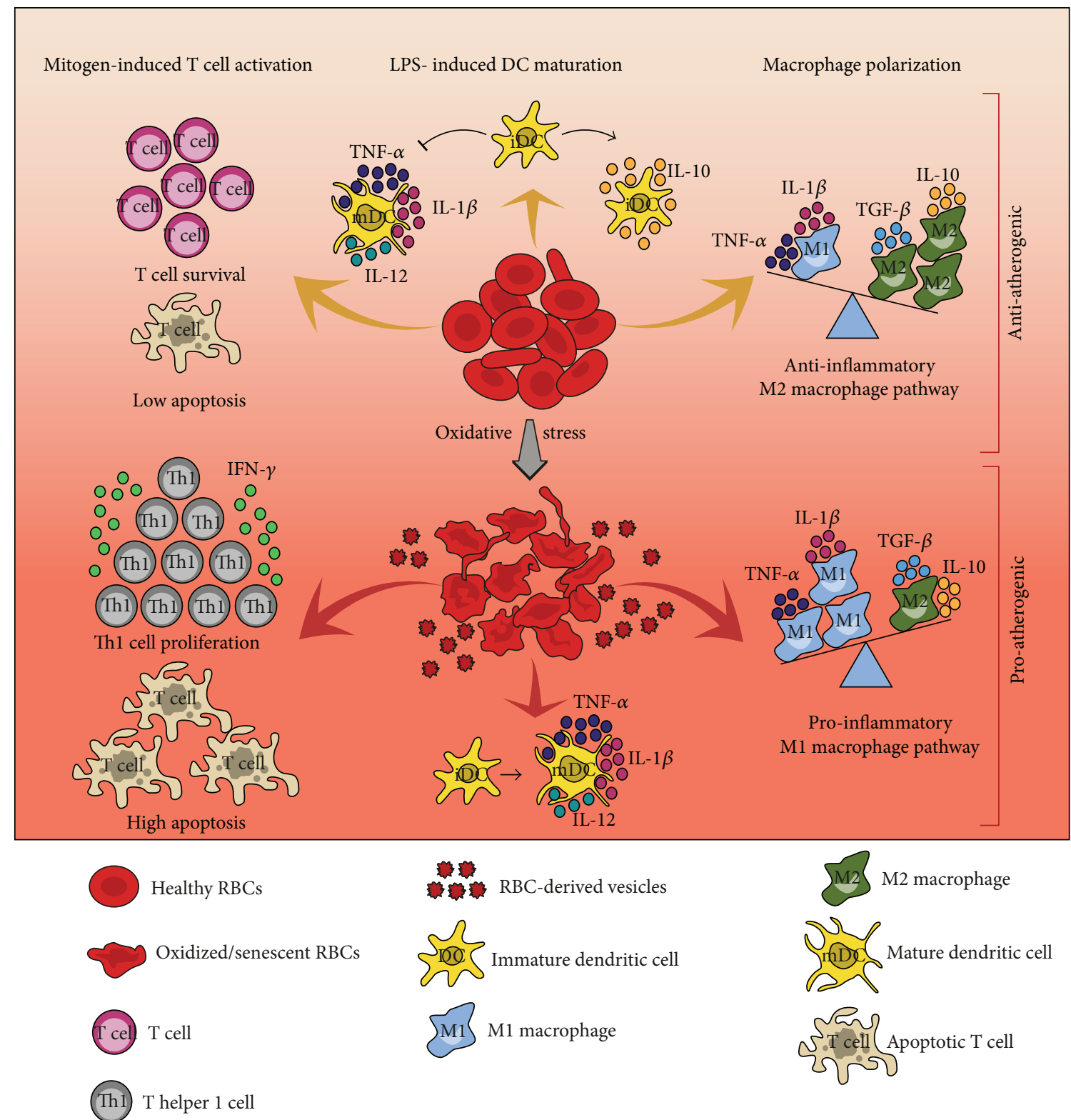

FIGURE 1: Proposed schematic model showing how the crosstalk between healthy or oxidized/senescent red blood cells (RBCs) and immune cells may exert anti- and proatherogenic effects, respectively. Human healthy RBCs release protein factors with the capacity to sustain T cell survival and to inhibit activation-induced T cell apoptosis. Healthy RBCs are also able to prevent a complete DC maturation in response to LPS, thus inducing phenotypic and functional characteristics typical of immature/tolerogenic DCs characterized by high IL-10 production. Healthy RBCs under noninflammatory conditions might promote a shift toward the anti-inflammatory M2 macrophage pathway. Thus, healthy RBCs may exert an immunomodulatory activity sustaining anti-inflammatory and antiatherogenic mechanisms. Under oxidative stress or store banking conditions, RBCs acquire an oxidized/senescent phenotype resulting in surface antigen modification or release of extracellular vesicles. In this way, oxidized/senescent RBCs or RBC-derived vesicles are capable of augment mitogen-driven T cell proliferation and apoptosis and determine a T helper 1 (Th1) proinflammatory and proatherogenic cytokine response. Oxidized/senescent RBCs, failing to control LPS-induced DC maturation, promote DC maturation toward a DC profile capable of inciting a proinflammatory Th1 cell response. Stored RBCs might polarize macrophages toward the classical M1 macrophage activation pathway associated with proinflammatory cytokine production. We propose that the oxidative injuries on RBCs likely represent dangerous signals for innate and adaptive immune cells, thus contributing or even triggering a damaging process worsening atherosclerotic disease.

\section{Crosstalk between Red Blood Cells and Innate and Adaptive Immune Cells}

3.1. Crosstalk between Red Blood Cells and Dendritic Cells. Several studies have implicated the role of DCs in progression and destabilization of the atherosclerotic plaque [58-61]. Dendritic cells are key sentinel cells of the innate immune system that possess the ability to stimulate the adaptive immunity $[62,63]$. During this process, activated DCs undergo distinct changes in phenotype and function, termed 
DC maturation $[62,64]$. This process involves a redistribution of major histocompatibility complex molecules, an increase in the surface expression of costimulatory molecules, morphological changes, secretion of chemokines, cytokines, and proteases, and surface expression of adhesion molecules and chemokine receptors. A variety of factors can induce DC maturation toward a DC profile capable of inciting primary T cell responses, including lipopolysaccharide (LPS), inflammatory cytokines, ligation of selected cell surface receptors, and viral products [62]. Conversely, several growth factors and cytokines including IL-10 modulate DC maturation, thus favouring the differentiation of tolerogenic DCs, DCs devoted to the maintenance of immunologic tolerance [62].

Schäkel and colleagues [2] have shown in vitro that an excess of RBCs, mimicking the physiological conditions in the blood, completely prevents the phenotypical maturation of the proinflammatory subset of circulating DCs (6-sulfo LacNAc+ DCs). The same study shows that RBCs are able to control interleukin (IL)-12 and tumor necrosis factor-alpha (TNF- $\alpha$ ) production of circulating DCs in response to LPS. The expression of CD47 on RBCs and that of inhibitory receptor signal regulatory protein alpha on circulating DCs appear to be critical for this inhibition [2].

Starting from the concept that in vitro senescent or oxidized or eryptotic RBCs modulate surface antigens [65, 66] and that these changes may significantly affect homotypic (RBC-RBC) and heterotypic (e.g., RBC-endothelial) interactions, thus altering RBC functional features [67], our research group has investigated whether RBCs from patients with carotid atherosclerosis maintain their immunomodulatory activity on human monocyte-derived DCs [16]. In this study, we confirmed that human healthy RBCs are able to prevent a complete DC maturation in response to LPS, thus inducing phenotypic and functional characteristics typical of tolerogenic DCs and characterized by reduced CD83, human leukocyte antigen-DR (HLA-DR), CD80, and CD86 surface expression associated with low IL-12, IL-6, and TNF$\alpha$ and high IL-10 production. In the same study, we also provided the first evidence that RBCs from patients with carotid atherosclerosis, unlike those from healthy subjects, fail to control LPS-induced DC maturation. LPS-stimulated DC cultured in the presence of patient RBCs presented a fully mature phenotype characterized by the upregulation of the surface molecules CD83, CD80, HLA-DR, and CD86 and by production of higher levels of proinflammatory cytokines. These results indicate that RBCs from patients with carotid atherosclerosis have an impaired immunomodulatory activity on DC functions, most likely due to the oxidized/senescent RBC phenotype [16]. The altered expression of CD47 at erythrocyte surface or its loss due to vesiculation could represent the main mechanism determining the functional impairment of patient erythrocytes in their crosstalk with DCs.

3.2. Crosstalk between Red Blood Cells and Macrophages. In the microenvironment of atherosclerotic plaques, the macrophage polarization state may accelerate or decelerate atherosclerotic disease progression, via activation or attenuation of inflammatory responses. However, the mechanisms underlying macrophage polarization and activation within the plaque remain unclear $[68,69]$. The interactions between macrophages and RBCs are important for RBC clearance and homeostasis [57]. In the liver and in the spleen, residential macrophages scrutinize passing RBCs and remove from the circulation those that are at the end of their lifespan or have sustained damage beyond repair [57]. At present there is no consensus as to how macrophages determine which RBCs need to be cleared and which ones can be repaired and/or maintained. Taking into account that RBCs are unable to synthesize new proteins, all "removal" markers must derive from modifications in preexisting molecules or to the acquisition of plasma-derived opsonins directed against these modifications. Furthermore, new evidence suggests that experimental aging of RBC induces a conformational change in CD47 that switches the molecule from an inhibitory into an activating one [70]. Although RBCs do not undergo classical apoptosis since they do not contain a nucleus, mitochondria, or other cellular organelles, the process they undergo has already been termed "eryptosis" since it exhibits many similarities with programmed cell death [71].

After intraplaque hemorrhage, a bulk of RBCs is deposited and degraded over days by macrophage phagocytosis activity through PS-receptors or natural occurring antibodies toward Band 3 or CD47-SIRP $\alpha$ interaction [72-75] as a part of a defence mechanism against oxidative burden, and this event promotes atherosclerotic lesion instability $[7,76]$. It is highly likely that phagocytosis of senescent or eryptotic RBCs in homeostatic conditions is noninflammatory [77]. The effect of RBC phagocytosis on cytokine secretion in inflammatory condition remains to be elucidated. Another point which needs to be addressed is whether macrophages participate in the resolution of plaque angiogenesis (that likely was the cause of hemorrhage) or actively contribute to it [78].

Yazdanbakhsh et al. [79] have proposed an interesting hypothetical model to explain the effect of stored versus fresh blood transfusion on macrophage plasticity. In this study they analyzed the $\mathrm{RBC}$ effects as response to $\mathrm{Hb}$ and the $\mathrm{Hb}$ breakdown product heme on macrophages. Macrophage plasticity is viewed as a spectrum of activation states ranging from the classic proinflammatory (M1) state, which is induced by the Thl cytokine interferon- (IFN-) $\gamma$ and bacterial components such as LPS, and the alternatively activated (M2) state, which is associated with the resolution phase of inflammation and driven by IL-4, IL-10, transforming growth factor beta, and glucocorticoids [80]. In a murine RBC storage and transfusion model, Hod et al. [81] showed that the transfusion of RBCs after prolonged storage induced macrophages to polarize toward the classical M1 macrophage activation pathway associated with bactericidal activity and proinflammatory cytokine production conducive of immunostimulation. The transfusion of fresh blood under noninflammatory conditions is associated with less RBC clearance [81] and therefore less loading of macrophages with heme, as well as with upregulation of heme oxygenase and a shift toward the M2 differentiation pathway, which is 
associated with immunoregulation through the induction of regulatory T cells [79].

During the lifespan of RBCs a loss of haemoglobin and membrane parts occurs from intact RBCs in vivo. In a rat model it has been shown that RBC-derived vesicles are rapidly removed from the circulation mainly by liver Kupffer cells and to a lesser extent by other macrophages of the mononuclear phagocyte system through scavenger receptors [82].

This evidence calls for in vivo and in vitro studies to verify the role of the oxidized, senescent RBCs or RBC-derived vesicles on macrophage polarization within haemorrhagic atherosclerotic lesions. The crosstalk between macrophages and RBCs might represent a potentially novel mechanism by which oxidative stress can contribute to atherosclerotic disease progression.

\subsection{Crosstalk between Red Blood Cells and T Lymphocytes.}

The binding of circulating immune cells to the vascular wall is a central process in inflammation, metastasis, and therapeutic cell delivery. In vivo and in vitro studies show that RBCs facilitate the engagement of circulating lymphocytes within the vascular endothelium $[15,83]$. It is well known that during inflammation and local immune response, capillary diameter and blood flow increase. This may allow the extravasation of RBCs, together with activated $\mathrm{T}$ cells, to the inflammatory place. A number of studies during the last three decades have presented evidence pointing to RBCs as putative modulators of $\mathrm{T}$ cell proliferation, both in vitro and in vivo [1]. In particular, RBCs are able to enhance $\mathrm{T}$ cell expansion and survival by inhibiting activation-induced $\mathrm{T}$ cell death, an effect possibly associated with a decrease of oxidative stress within activated $\mathrm{T}$ cells [14]. In previous studies optimal $\mathrm{T}$ cell proliferation and survival were only observed with intact RBCs and when RBCs were in close contact or proximity with activated $\mathrm{T}$ cells [14]. The need for RBC integrity and proximity might imply the involvement of interactions between receptors on each cell type, as suggested by some authors $[84,85]$. Recently it has been demonstrated that RBCs release protein factors with the capacity to sustain $\mathrm{T}$ cell growth and survival [13]. Our group demonstrated that RBCs from patients with carotid atherosclerosis maintain the ability to sustain in vitro the proliferative response of activated $\mathrm{T}$ lymphocytes [17]. Our results suggest that structural alterations and redox imbalance in patient RBCs do not prevent them from releasing some protein factors promoting $\mathrm{T}$ cell proliferation. Proliferating $\mathrm{T}$ cells in our study produced high concentrations of IFN- $\gamma$, the Thl cytokine involved in the inflammatory processes leading to plaque progression $[86,87]$. In contrast to us some studies suggest that RBCderived exosomes are potential modifiers of $\mathrm{T}$ cell responses in addition to their reported roles in innate immunity and $B$ cell responses $[88,89]$. The adverse patient outcomes associated with RBC storage length, observed in several clinical studies, well support our hypothesis on a pathogenic role of oxidized/senescent RBCs in complex inflammatory diseases such as atherosclerosis.

Stored RBCs, accompanied by shedding and release of extracellular vesicles, are capable of stimulating peripheral blood mononuclear cells (PBMC) in vitro and provoking proinflammatory cytokine response [41, 90]. Additionally, vesicle-derived RBCs augment mitogen-driven T cell proliferation in PBMC cultures in an antigen presenting cell-and cell contact-dependent manner [91, 92].

Apoptotic cell death has been demonstrated in advanced human atherosclerotic plaques [93] and impaired phagocytosis of apoptotic cells has been proposed as a mechanism that contributes to necrotic core formation to plaque vulnerability and rupture $[94,95]$. Our research group has showed that unlike RBCs obtained from healthy subjects, RBCs from patients and in vitro oxidized RBCs did not protect activated $\mathrm{T}$ lymphocytes from apoptosis [17]. Hence, RBCs from patients with carotid atherosclerosis, probably due to their oxidative imbalance, impact $\mathrm{T}$ cell integrity and function.

\section{Conclusion}

Collectively, literature data demonstrate that RBCs represent not only an efficacious mechanism to counteract oxidative stress but also a further tool to maintain immunological homeostasis. However, when an intense production of reactive species takes place, RBCs may acquire a prooxidant behavior [18] and lose their typical structural and functional features $[16-18,96]$. In particular, the oxidative injuries on RBC membrane, cytoskeleton, and cytoplasm components likely represent dangerous signals for the innate (e.g., DCs and macrophages) and adaptive immunity (e.g., T lymphocytes), thus contributing to or even triggering a damaging process worsening atherosclerotic disease. Improving our knowledge on the molecular basis of cellular interactions could offer opportunities for clinical intervention exploiting $\mathrm{RBC}$ and immune cell cooperation.

\section{Conflict of Interests}

The authors declare that there is no conflict of interests regarding the publication of this paper.

\section{Acknowledgment}

The authors thank Mr. Massimo Delle Femmine for the figures' graphic support.

\section{References}

[1] F. A. Arosa, C. F. Pereira, and A. M. Fonseca, "Red blood cells as modulators to T cell growth and survival," Current Pharmaceutical Design, vol. 10, no. 2, pp. 191-201, 2004.

[2] K. Schäkel, M. von Kietzell, A. Hänsel et al., "Human 6sulfo LacNAc-expressing dendritic cells are principal producers of early interleukin-12 and are controlled by erythrocytes," Immunity, vol. 24, no. 6, pp. 767-777, 2006.

[3] R. Businaro, A. Tagliani, B. Buttari et al., "Cellular and molecular players in the atherosclerotic plaque progression," Annals of the New York Academy of Sciences, vol. 1262, no. 1, pp. 134-141, 2012.

[4] G. K. Hansson and A. Hermansson, "The immune system in atherosclerosis," Nature Immunology, vol. 12, no. 3, pp. 204-212, 2011. 
[5] K. J. Woollard and F. Geissmann, "Monocytes in atherosclerosis: subsets and functions," Nature Reviews Cardiology, vol. 7, no. 2, pp. 77-86, 2010.

[6] R. Businaro, M. Digregorio, R. Riganò et al., "Morphological analysis of cell subpopulations within carotid atherosclerotic plaques," Italian Journal of Anatomy and Embryology, vol. 110, no. 2, pp. 109-115, 2005.

[7] A. P. Levy and P. R. Moreno, "Intraplaque hemorrhage," Current Molecular Medicine, vol. 6, no. 5, pp. 479-488, 2006.

[8] F. D. Kolodgie, H. K. Gold, A. P. Burke et al., "Intraplaque hemorrhage and progression of coronary atheroma," The New England Journal of Medicine, vol. 349, no. 24, pp. 2316-2325, 2003.

[9] P. Muda, P. Kampus, M. Zilmer et al., "Homocysteine and red blood cell glutathione as indices for middle-aged untreated essential hypertension patients," Journal of Hypertension, vol. 21, no. 12, pp. 2329-2333, 2003.

[10] E. Pytel, M. Olszewska-Banaszczyk, M. Koter-Michalak, and M. Broncel, "Increased oxidative stress and decreased membrane fluidity in erythrocytes of CAD patients," Biochemistry and Cell Biology, vol. 91, no. 5, pp. 315-318, 2013.

[11] T. Anuk, E. B. Assayag, R. Rotstein et al., "Prognostic implications of admission inflammatory profile in acute ischemic neurological events," Acta Neurologica Scandinavica, vol. 106, no. 4, pp. 196-199, 2002.

[12] B. N. Y. Setty, M. J. Stuart, C. Dampier, D. Brodecki, and J. L. Allen, "Hypoxaemia in sickle cell disease: biomarker modulation and relevance to pathophysiology," The Lancet, vol. 362, no. 9394, pp. 1450-1455, 2003.

[13] R. F. Antunes, C. Brandão, M. Maia, and F. A. Arosa, "Red blood cells release factors with growth and survival bioactivities for normal and leukemic T cells," Immunology and Cell Biology, vol. 89, no. 1, pp. 111-121, 2011.

[14] A. M. Fonseca, G. Porto, K. Uchida, and F. A. Arosa, "Red blood cells inhibit activation-induced cell death and oxidative stress in human peripheral blood T lymphocytes," Blood, vol. 97, no. 10, pp. 3152-3160, 2001.

[15] R. J. Melder, J. Yuan, L. L. Munn, and R. K. Jain, "Erythrocytes enhance lymphocyte rolling and arrest in vivo," Microvascular Research, vol. 59, no. 2, pp. 316-322, 2000.

[16] B. Buttari, E. Profumo, B. Cuccu et al., "Erythrocytes from patients with carotid atherosclerosis fail to control dendritic cell maturation," International Journal of Cardiology, vol. 155, no. 3, pp. 484-486, 2012.

[17] E. Profumo, B. Buttari, L. Petrone et al., "Redox imbalance of red blood cells impacts T lymphocyte homeostasis: implication in carotid atherosclerosis," Thrombosis and Haemostasis, vol. 106, no. 6, pp. 1117-1126, 2011.

[18] M. Minetti, L. Agati, and W. Malorni, "The microenvironment can shift erythrocytes from a friendly to a harmful behavior: pathogenetic implications for vascular diseases," Cardiovascular Research, vol. 75, no. 1, pp. 21-28, 2007.

[19] M. Comporti, C. Signorini, G. Buonocore, and L. Ciccoli, "Iron release, oxidative stress and erythrocyte ageing," Free Radical Biology and Medicine, vol. 32, no. 7, pp. 568-576, 2002.

[20] C. Signorini, M. Ferrali, L. Ciccoli, L. Sugherini, A. Magnani, and M. Comporti, "Iron release, membrane protein oxidation and erythrocyte ageing," FEBS Letters, vol. 362, pp. 165-170, 1995.
[21] F. Arslan, M. B. Smeets, B. Buttari et al., "Lack of haptoglobin results in unbalanced VEGF $\alpha$ /angiopoietin-1 expression, Intramural hemorrhage and impaired wound healing after myocardial infarction," Journal of Molecular and Cellular Cardiology, vol. 56, no. 1, pp. 116-128, 2013.

[22] B. Buttari, E. Profumo, R. Businaro et al., "Oxidized haemoglobin-driven endothelial dysfunction and immune cell activation: novel therapeutic targets for atherosclerosis," Current Medicinal Chemistry, vol. 20, no. 37, pp. 4806-4814, 2013.

[23] B. Buttari, E. Profumo, C. di Cristofano et al., "Haemoglobin triggers chemotaxis of human monocyte-derived dendritic cells: possible role in atherosclerotic lesion instability," Atherosclerosis, vol. 215, no. 2, pp. 316-322, 2011.

[24] R. P. Rother, L. Bell, P. Hillmen, and M. T. Gladwin, "The clinical sequelae of intravascular hemolysis and extracellular plasma hemoglobin: a novel mechanism of human disease," The Journal of the American Medical Association, vol. 293, no. 13, pp. 1653$1662,2005$.

[25] G. Silva, V. Jeney, Â. Chora, R. Larsen, J. Balla, and M. P. Soares, "Oxidized hemoglobin is an endogenous proinflammatory agonist that targets vascular endothelial cells," The Journal of Biological Chemistry, vol. 284, no. 43, pp. 29582-29595, 2009.

[26] P. W. Buehler, B. Abraham, F. Vallelian et al., "Haptoglobin preserves the CD163 hemoglobin scavenger pathway by shielding hemoglobin from peroxidative modification," Blood, vol. 113, no. 11, pp. 2578-2586, 2009.

[27] N. Leitinger, "Oxidized phospholipids as triggers of inflammation in atherosclerosis," Molecular Nutrition and Food Research, vol. 49, no. 11, pp. 1063-1071, 2005.

[28] G. Leonarduzzi, E. Chiarpotto, F. Biasi, and G. Poli, "4Hydroxynonenal and cholesterol oxidation products in atherosclerosis," Molecular Nutrition and Food Research, vol. 49, no. 11, pp. 1044-1049, 2005.

[29] J. H. Crawford, B. K. Chacko, C. G. Kevil, and R. P. Patel, "The red blood cell and vascular function in health and disease," Antioxidants \& Redox Signaling, vol. 6, no. 6, pp. 992-999, 2004.

[30] M. M. B. Kay, G. J. C. G. M. Bosman, G. J. Johnson, and A. H. Beth, "Band-3 polymers and aggregates, and hemoglobin precipitates in red cell aging," Blood Cells, vol. 14, no. 1, pp. 275289, 1988.

[31] A. Pantaleo, G. Giribaldi, F. Mannu, P. Arese, and F. Turrini, "Naturally occurring anti-band 3 antibodies and red blood cell removal under physiological and pathological conditions," Autoimmunity Reviews, vol. 7, no. 6, pp. 457-462, 2008.

[32] L. M. Snyder, F. Garver, S. C. Liu, L. Leb, J. Trainor, and N. L. Fortier, "Demonstration of haemoglobin associated with isolated, purified spectrin from senescent human red cells," British Journal of Haematology, vol. 61, no. 3, pp. 415-419, 1985.

[33] L. M. Snyder, L. Leb, J. Piotrowski, N. Sauberman, S. C. Liu, and N. L. Fortier, "Irreversible spectrin-haemoglobin crosslinking in vivo: a marker for red cell senescence," British Journal of Haematology, vol. 53, no. 3, pp. 379-384, 1983.

[34] H. U. Lutz and J. Fehr, "Total sialic acid content of glycophorins during senescence of human red blood cells," The Journal of Biological Chemistry, vol. 254, no. 22, pp. 11177-11180, 1979.

[35] F. E. Boas, L. Forman, and E. Beutler, "Phosphatidylserine exposure and red cell viability in red cell aging and in hemolytic anemia," Proceedings of the National Academy of Sciences of the United States of America, vol. 95, no. 6, pp. 3077-3081, 1998.

[36] T.-Y. Mao, L.-L. Fu, and J.-S. Wang, "Hypoxic exercise training causes erythrocyte senescence and rheological dysfunction by 
depressed Gardos channel activity," Journal of Applied Physiology, vol. 111, no. 2, pp. 382-391, 2011.

[37] G. J. C. G. M. Bosman, E. Lasonder, M. Luten et al., “The proteome of red cell membranes and vesicles during storage in blood bank conditions," Transfusion, vol. 48, no. 5, pp. 827-835, 2008.

[38] A. Stewart, S. Urbaniak, M. Turner, and H. Bessos, "The application of a new quantitative assay for the monitoring of integrin-associated protein CD47 on red blood cells during storage and comparison with the expression of CD47 and phosphatidylserine with flow cytometry," Transfusion, vol. 45, no. 9, pp. 1496-1503, 2005.

[39] R. L. Sparrow, G. Healey, K. A. Patton, and M. F. Veale, "Red blood cell age determines the impact of storage and leukocyte burden on cell adhesion molecules, glycophorin A and the release of annexin V," Transfusion and Apheresis Science, vol. 34, no. 1, pp. 15-23, 2006.

[40] A. B. Zimrin and J. R. Hess, "Current issues relating to the transfusion of stored red blood cells," Vox Sanguinis, vol. 96, no. 2, pp. 93-103, 2009.

[41] C. F. Hogman and H. T. Meryman, "Storage parameters affecting red blood cell survival and function after transfusion," Transfusion Medicine Reviews, vol. 13, no. 4, pp. 275-296, 1999.

[42] A. Giovannetti, L. Gambardella, D. Pietraforte et al., "Red blood cell alterations in systemic sclerosis: a pilot study," Cellular Physiology and Biochemistry, vol. 30, no. 2, pp. 418-427, 2012.

[43] E. Straface, L. Gambardella, A. Mattatelli et al., "The red blood cell as a gender-associated biomarker in metabolic syndrome: a pilot study," International Journal of Cell Biology, vol. 2011, Article ID 204157, 7 pages, 2011.

[44] E. Ingelsson, M. J. Pencina, G. H. Tofler et al., "Multimarker approach to evaluate the incidence of the metabolic syndrome and longitudinal changes in metabolic risk factors: the Framingham Offspring Study," Circulation, vol. 116, no. 9, pp. 984-992, 2007.

[45] D. Poli, E. Antonucci, E. Grifoni, R. Abbate, G. F. Gensini, and D. Prisco, "Gender differences in stroke risk of atrial fibrillation patients on oral anticoagulant treatment," Thrombosis and Haemostasis, vol. 101, no. 5, pp. 938-942, 2009.

[46] D. Konukoğlu, G. D. Kemerli, T. Sabuncu, and H. H. Hatemi, "Protein carbonyl content in erythrocyte membranes in type 2 diabetic patients," Hormone and Metabolic Research, vol. 34, no. 7, pp. 367-370, 2002.

[47] V. Yelinova, Y. Glazachev, V. Khramtsov, L. Kudryashova, V. Rykova, and R. Salganik, "Studies of human and rat blood under oxidative stress: changes in plasma thiol level, antioxidant enzyme activity, protein carbonyl content, and fluidity of erythrocyte membrane," Biochemical and Biophysical Research Communications, vol. 221, no. 2, pp. 300-303, 1996.

[48] N. S. Mangalmurti, S. Chatterjee, G. Cheng et al., "Advanced glycation end products on stored red blood cells increase endothelial reactive oxygen species generation through interaction with receptor for advanced glycation end products," Transfusion, vol. 50, no. 11, pp. 2353-2361, 2010.

[49] A.-M. Schmidt, M. Hofmann, A. Taguchi, S. D. Yan, and D. M. Stern, "RAGE: a multiligand receptor contributing to the cellular response in diabetic vasculopathy and inflammation," Seminars in Thrombosis and Hemostasis, vol. 26, no. 5, pp. 485493, 2000.

[50] B. Buttari, E. Profumo, A. Capozzi et al., "Advanced glycation end products of human $\beta_{2}$ glycoprotein I modulate the maturation and function of DCs," Blood, vol. 117, no. 23, pp. 6152-6161, 2011.
[51] B. Buttari, E. Profumo, F. Facchiano et al., "Resveratrol prevents dendritic cell maturation in response to advanced glycation end products," Oxidative Medicine and Cellular Longevity, vol. 2013, Article ID 574029, 12 pages, 2013.

[52] A. M. Schmidt and D. Stern, "Atherosclerosis and diabetes: the RAGE connection," Current Atherosclerosis Reports, vol. 2, no. 5, pp. 430-436, 2000.

[53] M. Ghashghaeinia, J. C. A. Cluitmans, A. Akel et al., "The impact of erythrocyte age on eryptosis," British Journal of Haematology, vol. 157, no. 5, pp. 606-614, 2012.

[54] M. H. Antonelou, A. G. Kriebardis, K. E. Stamoulis, E. Economou-Petersen, L. H. Margaritis, and I. S. Papassideri, "Red blood cell aging markers during storage in citrate-phosphatedextrose-saline-adenine-glucose-mannitol," Transfusion, vol. 50, no. 2, pp. 376-389, 2010.

[55] R. B. Ami, G. Barshtein, D. Zeltser et al., "Parameters of red blood cell aggregation as correlates of the inflammatory state," The American Journal of Physiology-Heart and Circulatory Physiology, vol. 280, no. 5, pp. H1982-H1988, 2001.

[56] S. Berliner, O. Rogowski, S. Aharonov et al., "Erythrocyte adhesiveness/aggregation: a novel biomarker for the detection of low-grade internal inflammation in individuals with atherothrombotic risk factors and proven vascular disease," American Heart Journal, vol. 149, no. 2, pp. 260-267, 2005.

[57] R. E. Mebius and G. Kraal, "Structure and function of the spleen," Nature Reviews Immunology, vol. 5, no. 8, pp. 606-616, 2005.

[58] Z. Mallat, H. Ait-Oufella, and A. Tedgui, "Regulatory TCell Immunity in atherosclerosis," Trends in Cardiovascular Medicine, vol. 17, no. 4, pp. 113-118, 2007.

[59] B. Østerud and E. Bjørklid, "Role of monocytes in atherogenesis," Physiological Reviews, vol. 83, no. 4, pp. 1069-1112, 2003.

[60] S. Ranjit and L. Dazhu, "Potential role of dendritic cells for progression of atherosclerotic lesions," Postgraduate Medical Journal, vol. 82, no. 971, pp. 573-575, 2006.

[61] A. Yilmaz, M. Lochno, F. Traeg et al., "Emergence of dendritic cells in rupture-prone regions of vulnerable carotid plaques," Atherosclerosis, vol. 176, no. 1, pp. 101-110, 2004.

[62] R. M. Steinman, "The dendritic cell system and its role in immunogenicity," Annual Review of Immunology, vol. 9, pp. 271296, 1991.

[63] R. M. Steinman, "The control of immunity and tolerance by dendritic cells," Pathologie Biologie, vol. 51, no. 2, pp. 59-60, 2003.

[64] H. Ueno, E. Klechevsky, R. Morita et al., "Dendritic cell subsets in health and disease," Immunological Reviews, vol. 219, no. 1, pp. 118-142, 2007.

[65] P. Matarrese, E. Straface, D. Pietraforte et al., "Peroxynitrite induces senescence and apoptosis of red blood cells through the activation of aspartyl and cysteinyl proteases," FASEB Journal, vol. 19, no. 3, pp. 416-418, 2005.

[66] D. Pietraforte, P. Matarrese, E. Straface et al., "Two different pathways are involved in peroxynitrite-induced senescence and apoptosis of human erythrocytes," Free Radical Biology and Medicine, vol. 42, no. 2, pp. 202-214, 2007.

[67] S. Yedgar, T. Hovav, and G. Barshtein, "Red blood cell intercellular interactions in oxidative stress states," Clinical Hemorheology and Microcirculation, vol. 21, no. 3-4, pp. 189-193, 1999.

[68] B. Buttari, E. Profumo, L. Segoni et al., "Resveratrol counteracts inflammation in human M1 and M2 macrophages upon challenge with 7-oxo-cholesterol: potential therapeutic implications 
in atherosclerosis," Oxidative Medicine and Cellular Longevity, vol. 2014, Article ID 257543, 12 pages, 2014.

[69] B. Buttari, L. Segoni, E. Profumo et al., "7-Oxo-cholesterol potentiates pro-inflammatory signaling in human M1 and M2 macrophages," Biochemical Pharmacology, vol. 86, no. 1, pp. 130-137, 2013.

[70] D. Z. de Back, E. B. Kostova, M. van Kraaij, T. K. van den Berg, and R. van Bruggen, "Of macrophages and red blood cells; A complex love story," Frontiers in Physiology, vol. 5, article 9, 2014.

[71] K. S. Lang, P. A. Lang, C. Bauer et al., "Mechanisms of suicidal erythrocyte death," Cellular Physiology and Biochemistry, vol. 15, no. 5, pp. 195-202, 2005.

[72] P. Arese, F. Turrini, and E. Schwarzer, "Band 3/complementmediated recognition and removal of normally senescent and pathological human erythrocytes," Cellular Physiology and Biochemistry, vol. 16, no. 4-6, pp. 133-146, 2005.

[73] P. Burger, P. Hilarius-Stokman, D. De Korte, T. K. van Den Berg, and R. van Bruggen, "CD47 functions as a molecular switch for erythrocyte phagocytosis," Blood, vol. 119, no. 23, pp. 5512-5521, 2012.

[74] M. Kay, "Immunoregulation of cellular life span," Annals of the New York Academy of Sciences, vol. 1057, pp. 85-111, 2005.

[75] H. U. Lutz, "Innate immune and non-immune mediators of erythrocyte clearance," Cellular and Molecular Biology (Noisyle-Grand), vol. 50, no. 2, pp. 107-116, 2004.

[76] R. Virmani, F. D. Kolodgie, A. P. Burke et al., "Atherosclerotic plaque progression and vulnerability to rupture: angiogenesis as a source of intraplaque hemorrhage," Arteriosclerosis, Thrombosis, and Vascular Biology, vol. 25, no. 10, pp. 2054-2061, 2005.

[77] A. E. Morelli, A. T. Larregina, W. J. Shufesky et al., "Internalization of circulating apoptotic cells by splenic marginal zone dendritic cells: dependence on complement receptors and effect on cytokine production," Blood, vol. 101, no. 2, pp. 611-620, 2003.

[78] A. V. Finn and J. Narula, "Intraplaque hemorrhage: most dangerous is the wound that bleedeth inwardly," JACC: Cardiovascular Imaging, vol. 5, pp. 856-858, 2012.

[79] K. Yazdanbakhsh, W. Bao, and H. Zhong, "Immunoregulatory effects of stored red blood cells," Hematology/the Education Program of the American Society of Hematology, vol. 2011, pp. 466-469, 2011.

[80] D. M. Mosser and J. P. Edwards, "Exploring the full spectrum of macrophage activation," Nature Reviews Immunology, vol. 8, no. 12, pp. 958-969, 2008.

[81] E. A. Hod, N. Zhang, S. A. Sokol et al., "Transfusion of red blood cells after prolonged storage produces harmful effects that are mediated by iron and inflammation," Blood, vol. 115, no. 21, pp. 4284-4292, 2010.

[82] F. L. A. Willekens, J. M. Werre, J. K. Kruijt et al., "Liver Kupffer cells rapidly remove red blood cell-derived vesicles from the circulation by scavenger receptors," Blood, vol. 105, no. 5, pp. 2141-2145, 2005.

[83] L. L. Munn, R. J. Melder, and R. K. Jain, "Role of erythrocytes in leukocyte-endothelial interactions: mathematical model and experimental validation," Biophysical Journal, vol. 71, no. 1, pp. 466-478, 1996.

[84] Y. Kalechman, S. Herman, U. Gafter, and B. Sredni, "Enhancing effects of autologous erythrocytes on human or mouse cytokine secretion and IL-2R expression," Cellular Immunology, vol. 148, no. 1, pp. 114-129, 1993.

[85] G. Virella, M. T. Rugeles, B. Hyman et al., "The interaction of CD2 with its LFA-3 ligand expressed by autologous erythrocytes results in enhancement of B cell responses," Cellular Immunology, vol. 116, no. 2, pp. 308-319, 1988.

[86] S. Taleb, A. Tedgui, and Z. Mallat, "Adaptive T cell immune responses and atherogenesis," Current Opinion in Pharmacology, vol. 10, no. 2, pp. 197-202, 2010.

[87] A. Tedgui and Z. Mallat, "Cytokines in atherosclerosis: pathogenic and regulatory pathways," Physiological Reviews, vol. 86, no. 2, pp. 515-581, 2006.

[88] S. Sadallah, C. Eken, and J. A. Schifferli, "Erythrocyte-derived ectosomes have immunosuppressive properties," Journal of Leukocyte Biology, vol. 84, no. 5, pp. 1316-1325, 2008.

[89] M. Schiller, M. Parcina, P. Heyder et al., "Induction of type I IFN is a physiological immune reaction to apoptotic cell-derived membrane microparticles," The Journal of Immunology, vol. 189, no. 4, pp. 1747-1756, 2012.

[90] D. B. Kim-Shapiro, J. Lee, and M. T. Gladwin, "Storage lesion: role of red blood cell breakdown," Transfusion, vol. 51, no. 4, pp. 844-851, 2011.

[91] W. Jy, M. Ricci, S. Shariatmadar, O. Gomez-Marin, L. H. Horstman, and Y. S. Ahn, "Microparticles in stored red blood cells as potential mediators of transfusion complications," Transfusion, vol. 51, no. 4, pp. 886-893, 2011.

[92] A. Danesh, H. C. Inglis, R. P. Jackman et al., "Exosomes from red blood cell units bind to monocytes and induce proinflammatory cytokines, boosting T-cell responses in vitro," Blood, vol. 123, no. 5, pp. 687-696, 2014.

[93] A. Tedgui and Z. Mallat, "Apoptosis as a determinant of atherothrombosis," Thrombosis and Haemostasis, vol. 86, no. 1, pp. 420-426, 2001.

[94] S. Björkerud and B. Björkerud, "Apoptosis is abundant in human atherosclerotic lesions, especially in inflammatory cells (macrophages and T cells), and may contribute to the accumulation of gruel and plaque instability," The American Journal of Pathology, vol. 149, no. 2, pp. 367-380, 1996.

[95] M. M. Kockx and M. W. M. Knaapen, "The role of apoptosis in vascular disease," The Journal of Pathology, vol. 190, no. 3, pp. 267-280, 2000.

[96] J. H. Crawford, B. K. Chacko, C. G. Kevil, and R. P. Patel, "The red blood cell and vascular function in health and disease," Antioxidants and Redox Signaling, vol. 6, no. 6, pp. 992-999, 2004. 


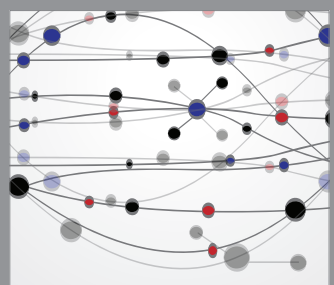

The Scientific World Journal
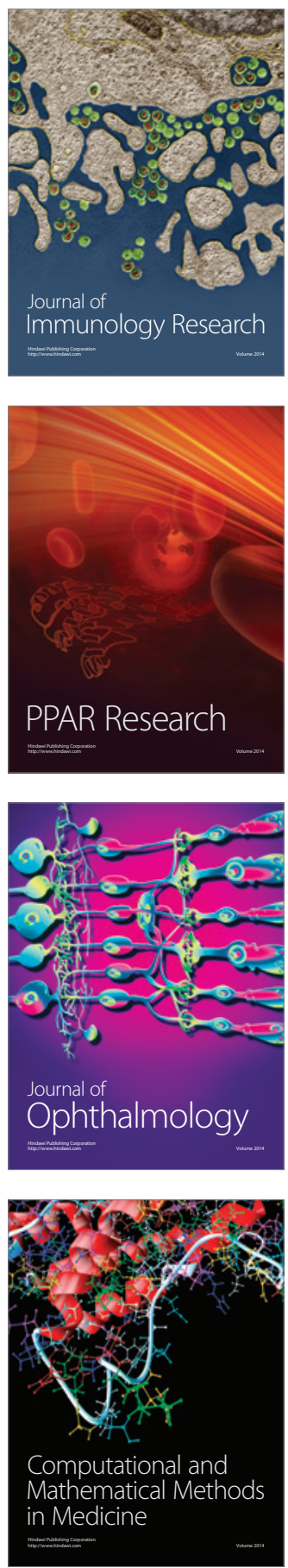

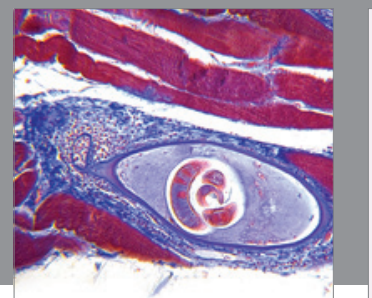

Gastroenterology

Research and Practice
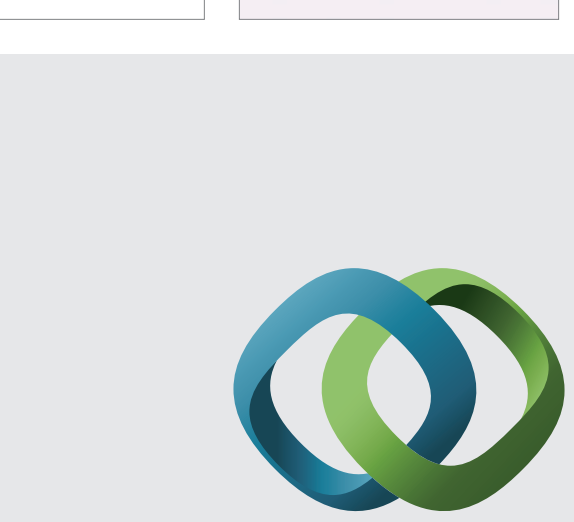

\section{Hindawi}

Submit your manuscripts at

http://www.hindawi.com
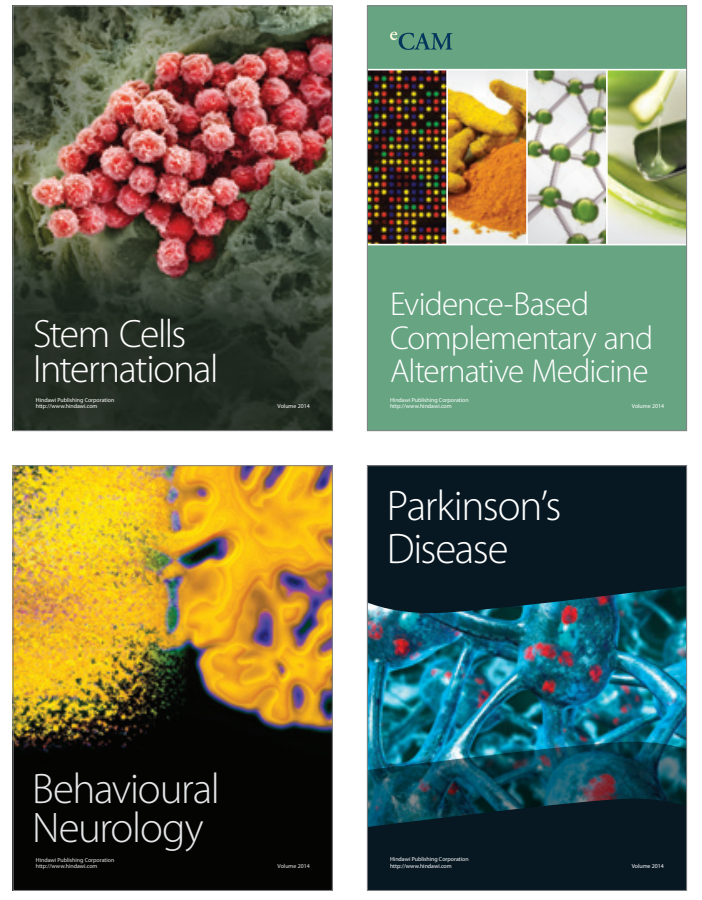
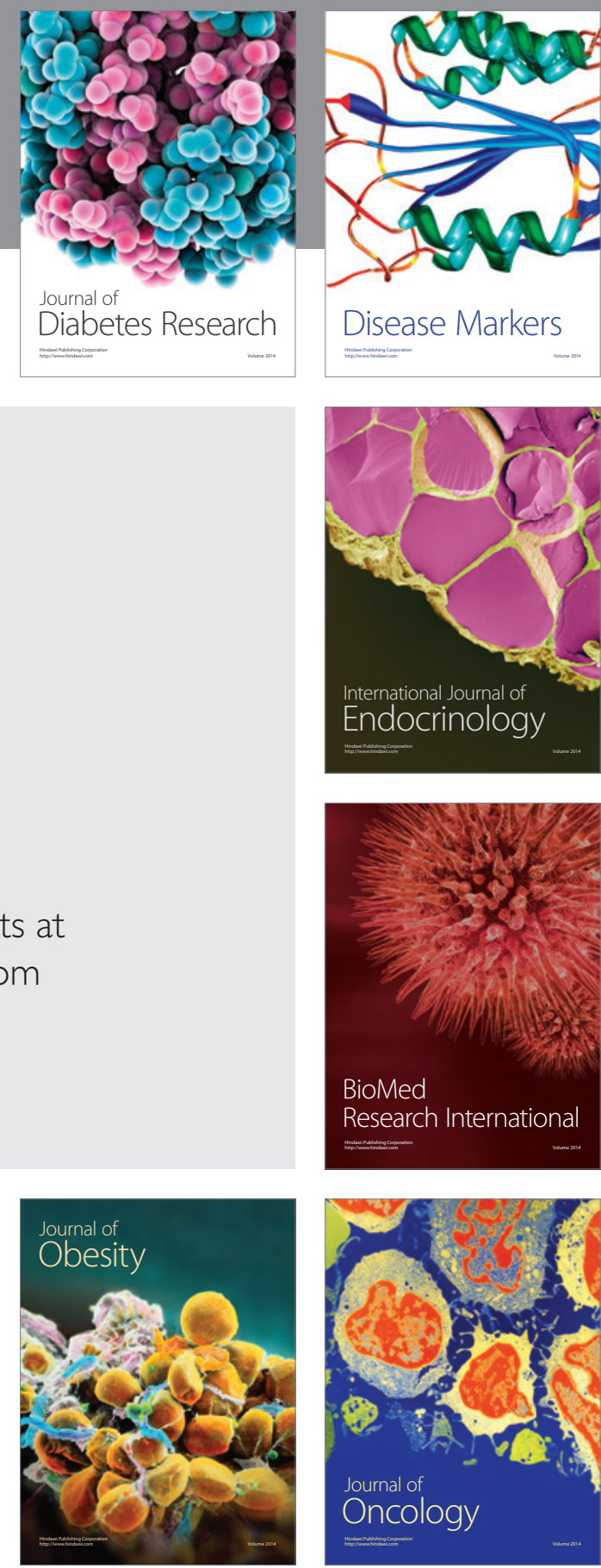

Disease Markers
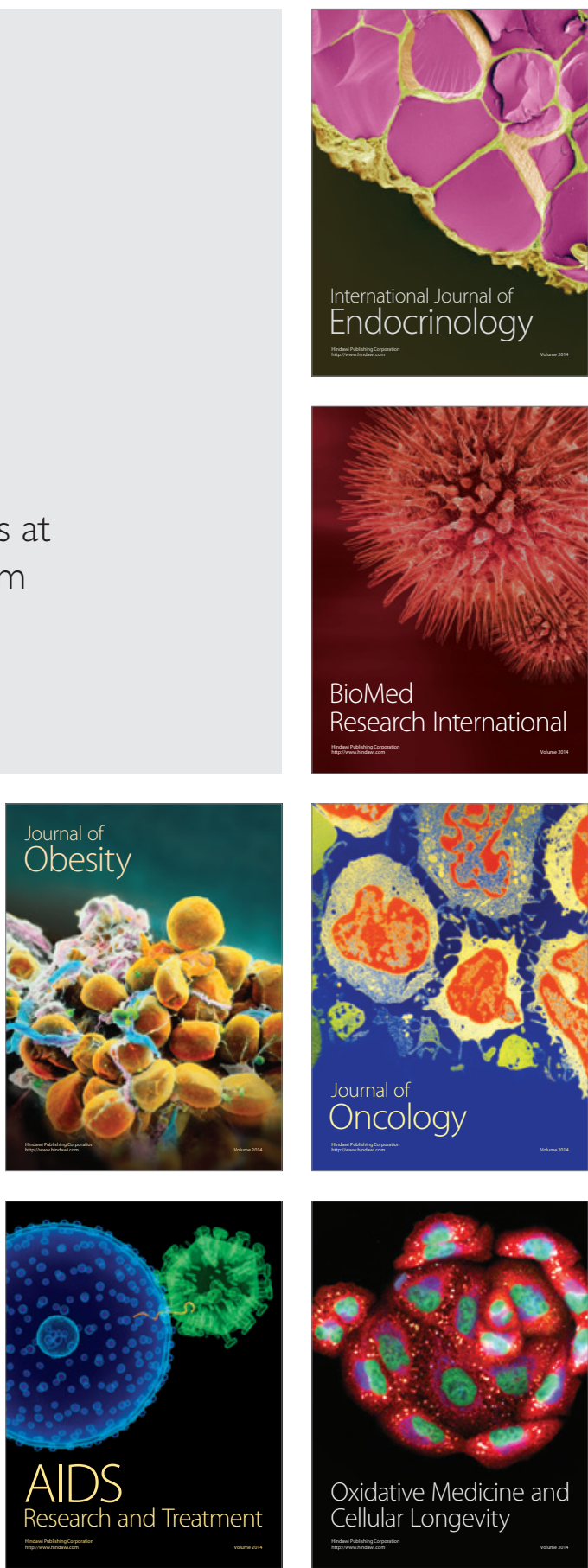